\title{
Finding stillness within the shaken brain
}

$\mathrm{N}$ o matter how hard I try, I cannot recall the moments just before I crashed on that September morning in 2007. It was my usual bike ride down the gravel path to work, when somehow, I fell, landing head first into the ground. A concerned jogger helped me down the hill, then my husband brought me to my busy urgent care clinic. Concussed and through a heavy haze, I saw patients until management could arrange a replacement. As I pushed through my duties, I recall the nurses commenting on my pale and drawn appearance. After a couple of hours, I could no longer continue. My head was not my own, and I felt overwhelmed by the numerous voices around me as I endeavoured to write my charts. I suppose, because of feelings of guilt, embarrassment or denial, I did not get "checked." Security arranged a taxi and I returned home to sleep, unobserved; not the usual approach to a concussed patient. In fact, it was a potentially dangerous oversight, considering that my father had died within 24 hours from an intracranial hemorrhage after a fall from his bicycle in 1991.

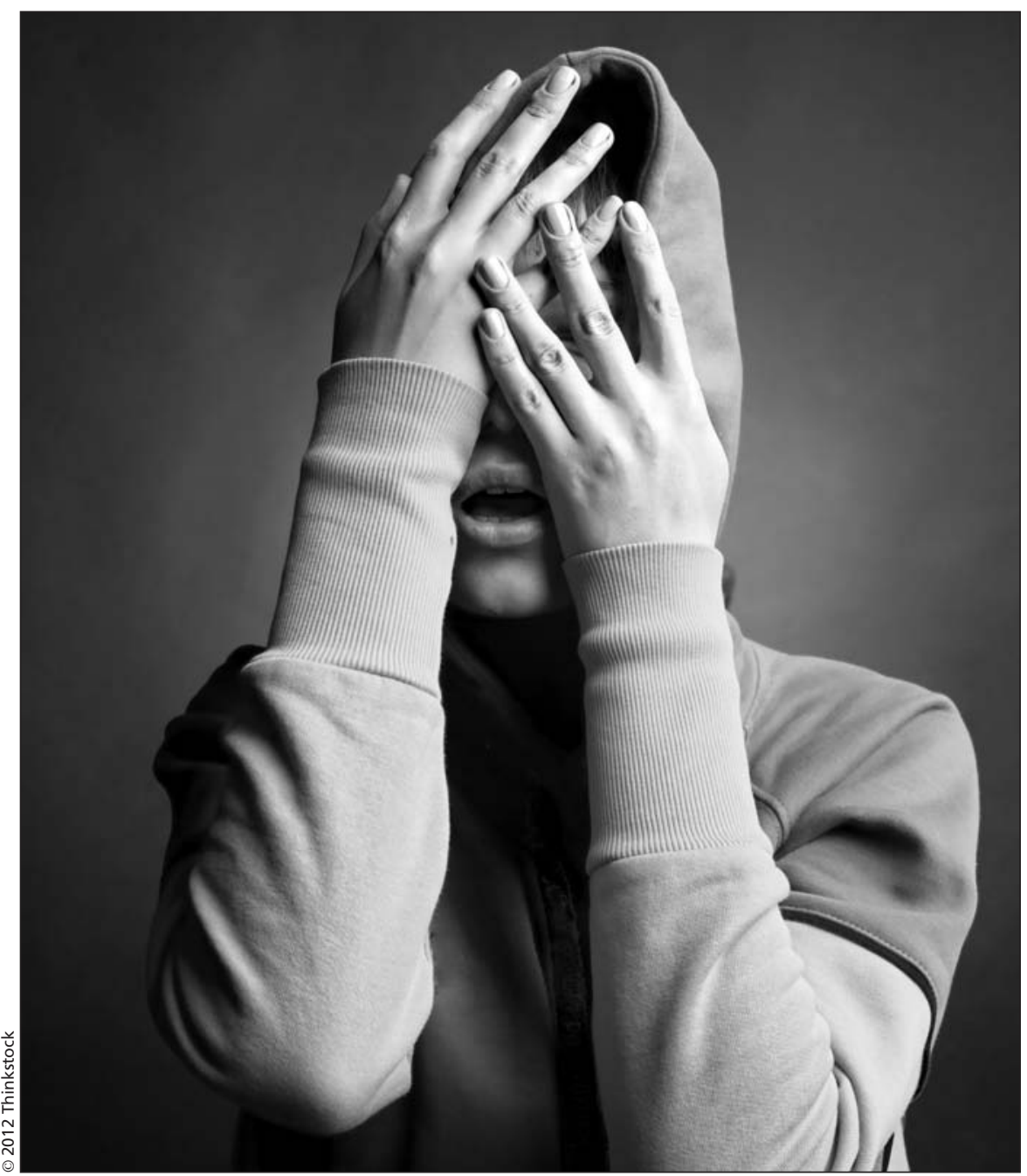

Over the weeks that followed, dizziness, head pressure and overwhelming input from noisy, bright environments kept me from working as a physician. My vision seemed the most affected by the concussion. I had difficulty focusing and tracking. Concentrating on a conversation took enormous effort, forcing me to look down to rest my eyes. The current concussion advice in 2007 was to "rest." However, for an active mother (of children ages 2 and 5), wife and an urgent care physician, "rest" was a relative term. After two weeks of "rest," a brief three-hour return to work resulted in extreme eye strain, nausea and dizziness. At that point, I was taken off work for two more months and sent home with no definitive plan. Thoughts of inadequacy and guilt weighed on my conscience regarding work. Knowing I was considered a danger to my patients embarrassed me as my cognitive abilities were now inadequate to practise medicine. Yet, being at home at "rest," did not change the expectation that I would still have to take on all the responsibilities of the house and child care with no extra help. How was that any different from work?

I tried and tried to keep up. My usual release from stress and insomnia through exercise had been taken away because it worsened symptoms. Adrenaline took over so I could meet the demands of home life. I presume this was my response to the trauma, by trying to overcompensate. As I grew more fatigued and my symptoms persisted, negative thoughts and frustration escalated. Although I looked normal to others, I wasn't. My shaken brain was slow and easily fatigued from processing the multitude of sensory information. I had an old person's brain - sensitive to light and sound - and the bobbling head of a child - moving with momentum but little coordination. My usual 3-D body awareness seemed rather impaired. 
Why didn't the doctors tell me I was so vulnerable? Two months after the first hit, I stood up in my kitchen, ramming my head into the corner of an open cupboard door. I collapsed, feeling dazed and nauseated with a new searing pain through my head. This time, I went to emergency. The head CT was "normal" as expected, but I felt "shell shocked" paralyzed by an overwhelming sense of panic and fatigue. Had I done something terrible to my brain? Was I going to be mentally impaired for the rest of my life?

At this point, I was in bed full time with photo- and phonosensitivity, insomnia and a constant migraine-like headache. I tried "rest" again. After a week and not wanting to disappoint my kids, the inconceivable happened ... I hit my head again on a cupboard above a school toilet. The third blow finished me. I couldn't cope with stress of any kind. I presented to the specialist, curled up into a little ball of pain, wearing my padded hat and dark glasses. At a loss, the specialist finally referred me to the minor brain injury clinic.

I lost all sense of control in my life. My body gave me no choice but to stop doing everything. I lay alone in my bed in the dark for about six months. The eye and head pain were unrelenting. I was now wearing a helmet, earplugs, and sunglasses in my house, which I only left for appointments. The posttraumatic stress anxiety was constant. I stopped listening to the radio after hearing about a wrestler who had murdered his family and killed himself after having numerous concussions. Although there were many other factors to his story, what resonated with me were his numerous concussions. I worried that it could happen to me. My appetite was nonexistent and I dropped 15 pounds. My husband was typically late getting to work, and would stay home with me when he sensed my courage was fading.

Our part-time nanny took over all meals, cleaning, child care, messages, opening mail, receiving visitors, chauffeuring and caring for her broken employer. My husband coped the best he could with our confused children and our uncertain future. My book club angels came weekly with meals and reassurance. Family and very close friends flew out individually to help. They all endured a house of darkness and forced silence as I slowly navigated the house, eyes closed. I identified individuals by the sound of their footsteps. I knew the button positions on the phone for the brief calls I could tolerate with the outside world. The hair on my body grew to Sasquatch proportions as it was too painful to focus my eyes to shave. My cat lying upon me was my constant companion. The worse my headache, the closer she would snuggle under my chin.

Over time, it became apparent that the lack of a plan and a team were critical omissions. The result was a rehabilitation path of trial and error. Each week, I could manage a couple of appointments to specialists: an expert in cranial sacral massage, social worker, psychologist, optometrist, physiatrist, physical therapist, headache neurologist, neuro-ophthalmologist, family doctor, visual therapist and concussion specialist. Most of these were selfreferred, some out of pure desperation. Unpredictably, the visual therapy exercises landed me back in bed for a further two months with migraine headaches. I battled with numerous medications and their individual side effects. Unable to focus, my endless worries included obsessing that our cat might be poisoned by the unfound portions of pills I so clumsily chopped in half.

I was blessed with a guardian angel in my cranial sacral massage therapist. Having had two concussions herself, she taught me breathing and relaxation. Slowly, I expanded my skills with meditation, yoga, pacing, visualization and self-management for chronic pain. My best progress came when I finally surrendered (different from giving up) and accepted my situation. My frustration and fear gradually dissolved and I learned to just "Be." This journey was mine and no one else could travel it. It wasn't good or bad, it just was. I began to see how my injury could become a gift. I nurtured the opportunity to retrain my brain. With eyes being my weakness, I started to listen. Audio books became my main form of education. As I improved, I experienced a form of euphoria. It spread through me each time I successfully retried an activity I hadn't done for two years. Shopping had this effect. Early on, attempts to scan the toothbrush aisle with all its small boxes in endless columns of colours and sizes were agonizing. I would retreat in painful defeat. Now, the idea of actually being able to buy something for myself was exciting. I was alive. It seemed I was being given a second chance to experience the world. The more I endeavoured and accomplished, the more I was able to rebuild my confidence and independence.

At the two-year mark, I started to drive again, read and briefly watch TV. My vision started to settle by the fifth eye glass prescription change and I graduated from two pairs of sunglasses to one. Sadly, I have had to grieve the loss of my career as an urgent care physician. Unfortunately, I still experience unpredictable visual auras with subsequent migraines, fatigue and mild sensory sensitivity. I am, however, much improved and I am so grateful. I can care for my family and others now. I see patients in a walk-in clinic where the staff scan my notes to the computer thereby easing the strain on my eyes.

My life has changed in so many ways. As a doctor, I have a much deeper understanding of the subtleties of illness. As a mother and partner, I am still learning to pace myself, set boundaries and prioritize. As an individual, I listen more to my heart and live life in the moment, trying not to be swept away by the influences of the busy world around me.

\section{Daphne L. Anderson MD \\ Calgary Sports Therapy \\ A facility of LifeMark Health \\ Calgary, Alta.}

Daphne has recently achieved the CASEM diploma exam and is setting up a practice in sports medicine. She has also submitted for publication her protocol that she uses with patients for the management of concussion, entitled "Brainrest": A Specific Detailed Approach to Cognitive Rest.

CMAJ 2012. DOI:10.1503/cmaj.120074 